Journal of Advanced Research in Fluid Mechanics and Thermal Sciences

\title{
Effect of Mesh Type on Numerical Computation of Aerodynamic Coefficients of NACA 0012 Airfoil
}

\author{
Mostafa Abobaker ${ }^{1}$, Sogair Addeep ${ }^{1}$, Lukmon O Afolabi², Abdulhafid M Elfaghi ${ }^{2,{ }^{*}}$ \\ 1 Department of Aeronautical Engineering, Faculty of Engineering, University of Zawia, Libya \\ 2 Faculty of Mechanical and Manufacturing Engineering, Universiti Tun Hussein Onn Malaysia, Batu Pahat, 86400I Johor, Malaysia
}

ARTICLE INFO ABSTRACT

\section{Article history:}

Received 27 July 2021

Received in revised form 1 September 2021

Accepted 5 September 2021

Available online 28 September 2021
Keywords:

Mesh Type; NACA 0012; Airfoil; CFD

\begin{abstract}
Mesh type and quality play a significant role in the accuracy and stability of the numerical computation. A computational method for two-dimensional subsonic flow over NACA 0012 airfoil at angles of attack from $0^{\circ}$ to $10^{\circ}$ and operating Reynolds number of $6 \times 10^{6}$ is presented with structured and unstructured meshes. Steady-state governing equations of continuity and momentum conservation are solved and combined with $k-w$ shear stress transport (SST-omega) turbulence model to obtain the flow. The effect of structured and unstructured mesh types on lift and drag coefficients are illustrated. Calculations are done for constant velocity and a range of angles of attack using Ansys Fluent CFD software. The results are validated through a comparison of the predictions and experimental measurements for the selected airfoil. The calculations showed that the structured mesh results are closer to experimental data for this airfoil and under studied operating conditions.
\end{abstract}

\section{Introduction}

Computational Fluid Dynamics (CFD) plays a vital role in nowadays routine design and analysis engineering work. CFD is one of the numerical methods of choice in the simulation and design of many aerospace, automotive and industrial components to reduce the number of wind tunnel testing and increase the number of design configurations being explored [1]. CFD starts with selecting the mathematical model that describes the problem, followed by specifying the computational domain of interest. Computational domain discretization or mesh generation is an early and essential step in almost all commercial numerical solution packages. Therefore, it is crucial to choose mesh type and size from the beginning of the solution process. Mesh generation can be classified into two general types structured and unstructured. Structured meshes are typically either quad or hexahedral. Triangle and Tetrahedral meshes are the most common unstructured meshes. As to the answer to the question, which is better. It is problem-dependent. Flow alignment (grid quality) and fewer nodes (memory and computation time) are in the fervor of structured grid. Unstructured grid types are more suitable with complex geometries and complex 3D shapes [2]. That is why it is simpler to

\footnotetext{
* Corresponding author.

E-mail address: abdulhafid@uthm.edu.my
}

https://doi.org/10.37934/arfmts.87.3.3139 
generate unstructured mesh for irregular shapes. Therefore, this paper aims to explore the discretization effect on aerodynamic lift and drag coefficients of an airfoil.

When simulating the flow over an airfoil, the effect of a grid on the solution convergence and accuracy is crucial to the accuracy and the computational cost of the simulation process. Grid generation consumes over $80 \%$ of the numerical simulation time [3]. Recently, Shu least squarebased finite difference-finite volume (LSFD-FV) method on unstructured grids was proposed by Shu and his colleagues [4-6]. Kajal et al., worked on meshing over an airfoil design with a linear triangle element, keeping the idea of meshing intuitive [7].

Flow around bluff bodies using structured grids for numerical simulation are presented in many letratures $[8,9]$. The generation of organized grids is a bigger influence for complex shape domains in the computational phase. However, the unstructured grids should be running more effectively and more flexibly. In hydrodynamics, unstructured triangular grids are widely used in practical engineering. The gradient smoothing method (GSM) solution with unstructured triangular grids is stable and precise, according to repeated research efforts. Therefore, GSM has been applied to solve a series of practicing solids and fluids flows governed by the Lagrangian Navier-Stokes equations. [1012].

Among others, the Large-Eddy Simulation (LES) structured mesh of high Reynolds number flow past a square cylinder is presented by Sohankar [13] and Voke [14]; and more recently by Mao and Liu [15]. LES applications in real-world complex configurations, such as the actual urban environment and full-scale bridges, are becoming the earliest convenience in order to bring numerical predictions closer to reality. These complex configurations allow un-structured grids that are defined by irregular connectivity to be implemented. The possible elements can be of arbitrary form. The numerical schemes of second order precision are still widely used on unstructured grids by most generalpurpose computational fluid dynamics (CFD) codes, including the major commercial solvers. Structured meshing is employed by Khan et al., [16] to investigate for the analysis for lift and drag coefficients using different turbulence models. Hence, Abobaker et al., [17] applied unstructured grid for the two-dimensional subsonic flow over a NACA 0012 airfoil to investigate a wind tunnel wall effect on lift curve slope correction factor.

In this study, lift and drag characteristics of the NACA 0012 airfoil as a function of angle of attack are computed. This airfoil is selected because it offers a wide range of uses. The B-17 Flying Fortress and Cessna 152, the helicopter Sikorsky S-61 SH-3 Sea King, and horizontal and vertical axis wind turbines are also examples of airfoil used. The Reynolds-averaged Navier-Stokes equations are solved inside the computational domain to yield the aerodynamic lift and drag coefficients. The details of the mathematical model can be found in many references [18] and [19] are avoided, and its numerical solution is given elsewhere. In order to evaluate the aerodynamics characteristics of the NACA 0012 airfoil were performed some CFD analyses in the following conditions, as in many experimental investigations in usual wind tunnels [20-22]. A Mach number 0.15 , Reynolds number of the flow is $6 \times 10^{6}$, and angle of attack range from 0 to 10 degrees. The airfoil chord length is $1.752 \mathrm{~m}$. The rest of the reference values were considered as the aviation standard for air.

\section{Structured Mesh}

A square domain is used for structured mesh with boundary conditions, as illustrated in Figure 1. The computational domain extends $12 \mathrm{C}$ in all directions, where $\mathrm{C}$ is the chord length. This is to ensure very far boundaries so we can simulate freestream conditions. A mesh sensitivity study is performed, which results in a number of node points in the entire computational domain of 154560 and the number of elements of 153600. A close-up view of the mesh is shown in Figure 2. 


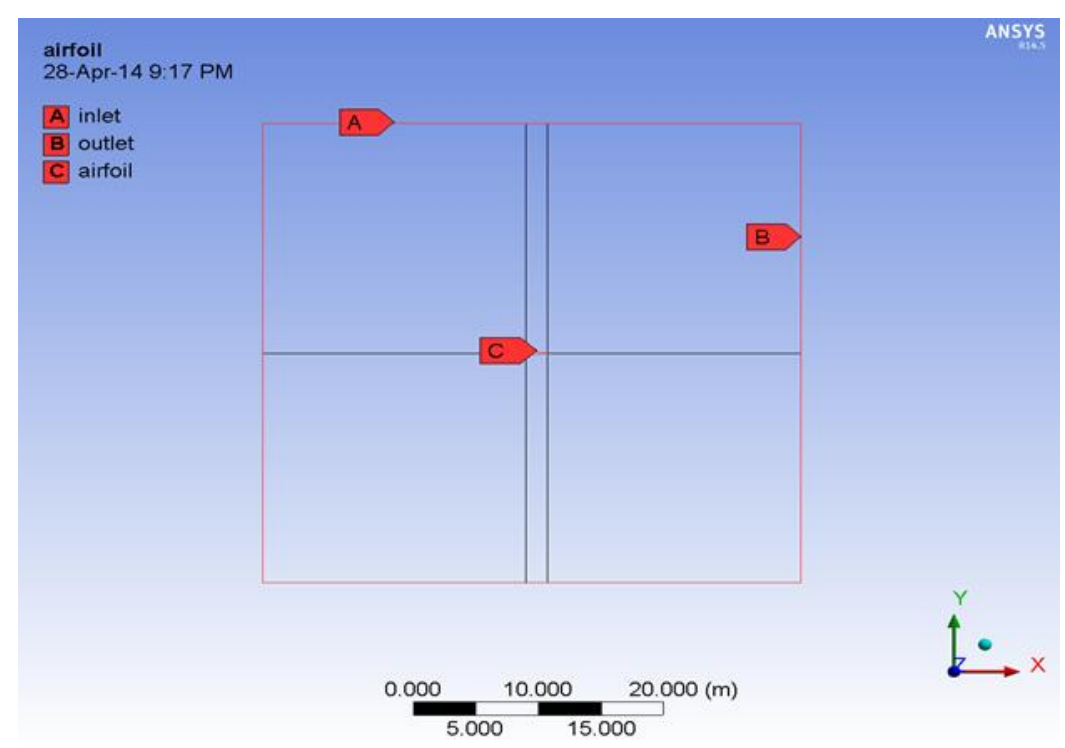

Fig. 1. A general view of the computational domain

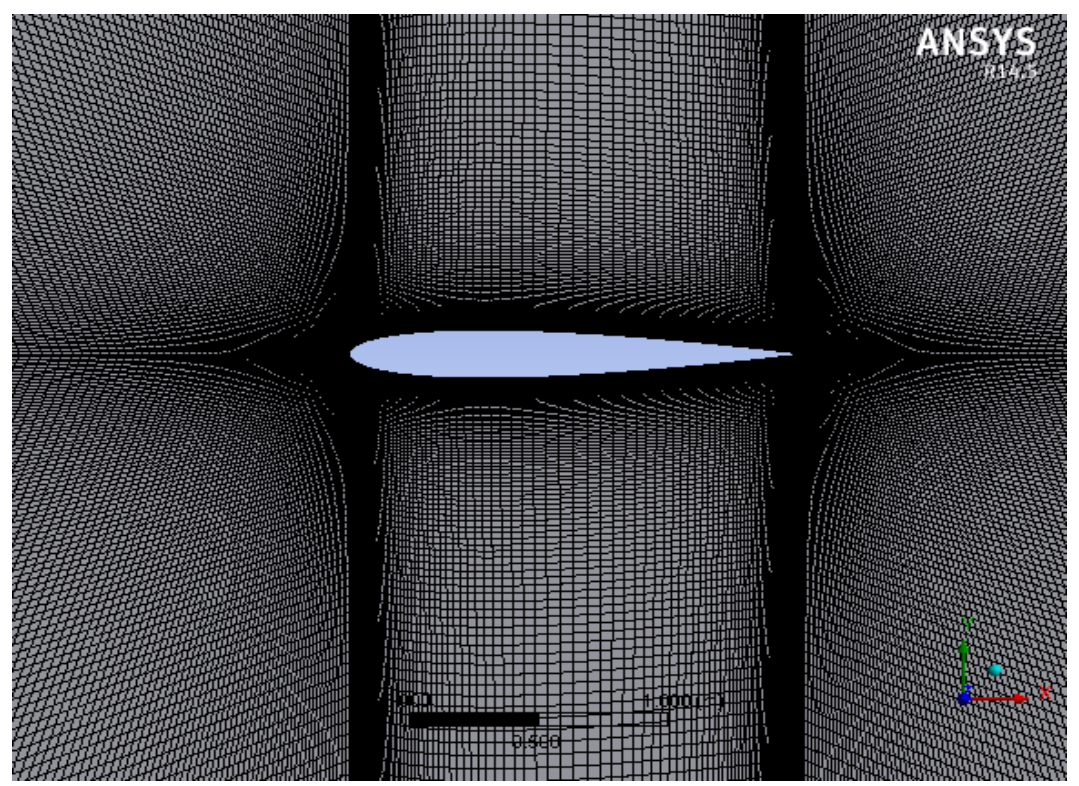

Fig. 2. A general view of the computational domain

The obtained results are presented graphically in Figure 3, and the drag polar in Figure 4. The obtained results are compared with the experimental data published in the literature [20-22]. K $\omega$ SST turbulence model is used for a range of angles of attack up to $12^{\circ}$.

Simulation results show that lift and drag coefficients have good agreement with experimental data up to the angle of attack $8^{\circ}$. It can be seen from Figure 3 that there is a significant difference between published data for angles of attack greater than $8^{\circ}$. On the other hand, simulation results are very close to the experimental data obtained from [20]. It is worth noting that these results were obtained by eliminating the high skewness from domain bounders mesh and clustering the mesh around leading and trailing edges of the airfoil. 


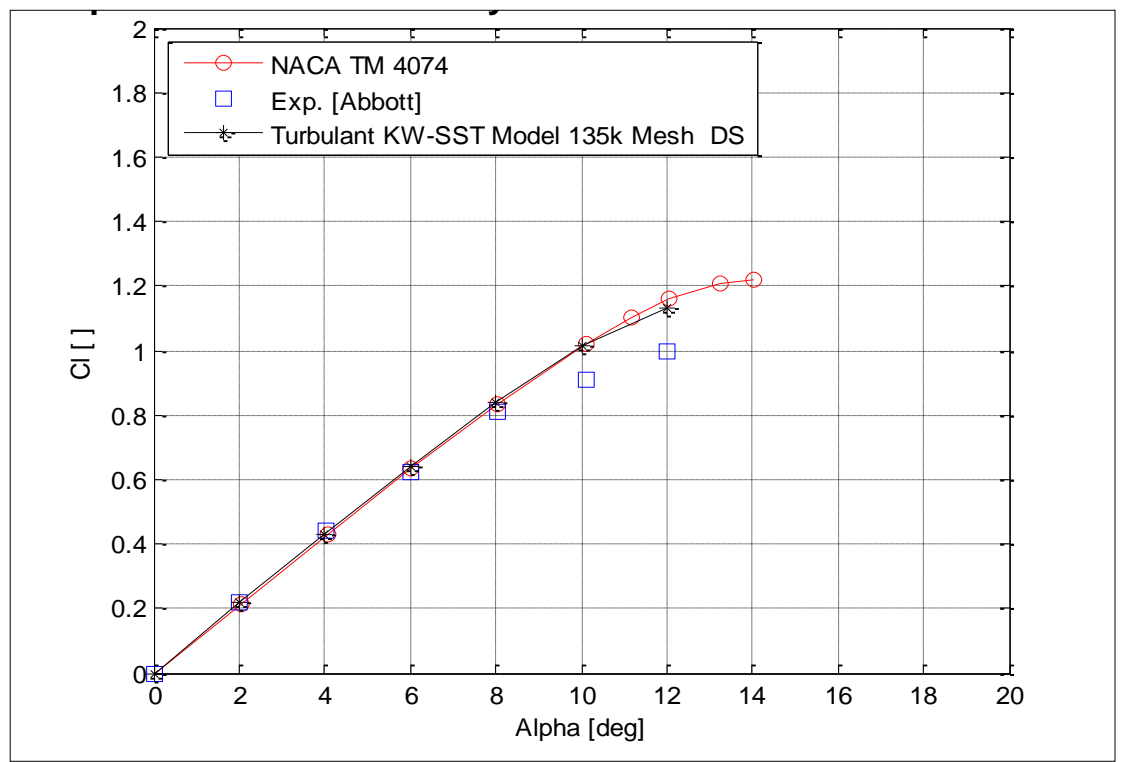

Fig. 3. Comparison of lift coefficient versus angle of attack with experimental data $[20,22]$

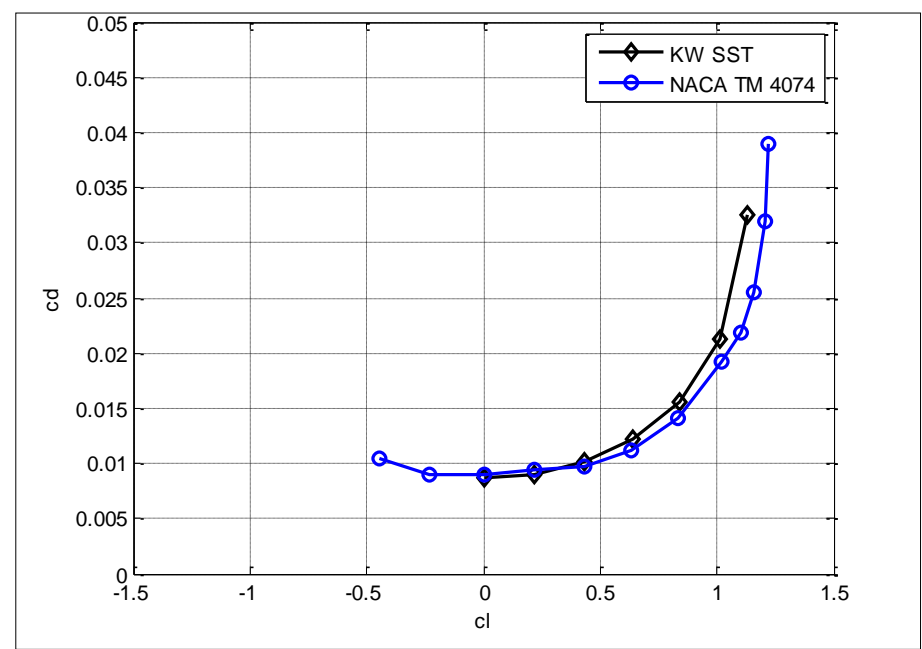

Fig. 4. Comparison of drag polar with experimental data $[21,22]$

\section{Unstructured Mesh}

A square domain is also utilized for the unstructured mesh, as shown in Figure 5 . The size is $12 \mathrm{C}$ in each direction. The number of node points is 12371 point and the number of elements is 23298 elements. A close-up view in Figure 6 shows a mesh clustering near the wall to capture boundary layer. 


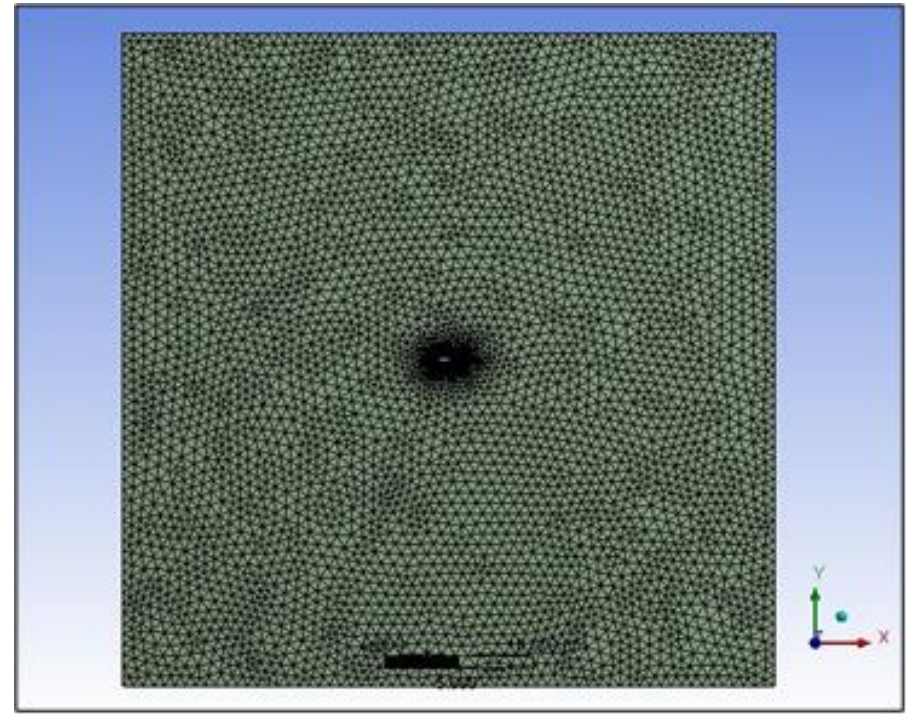

Fig. 5. A general view of the computational domain and the unstructured mesh

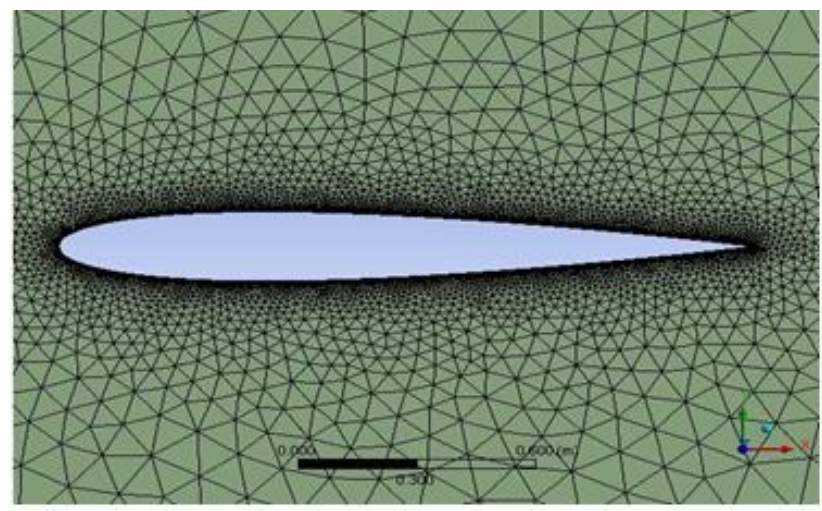

Fig. 6. A close-up view of the airfoil profile in unstructured rectangular grid

The obtained results of lift coefficient versus angle of attack and drag polar are presented in Figure 7 and Figure 8, respectively. The obtained results were compared with the experimental data. As can be seen, there are acceptable differences between results obtained with Fluent concerning the lift and drag coefficients. The deviations from experimental data between the angles of attack of $8^{\circ}$ to $10^{\circ}$ are acceptable.

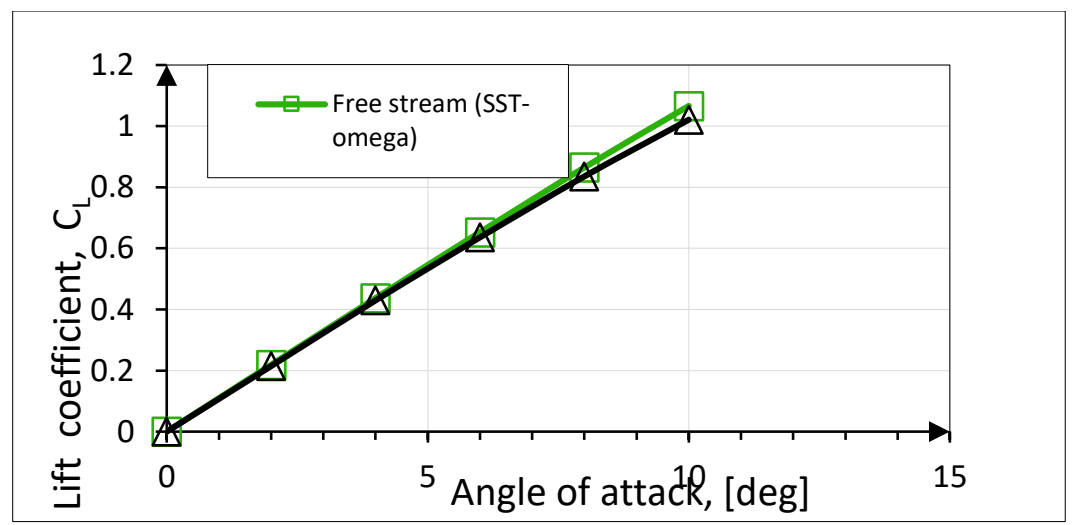

Fig. 7. Comparison of calculated lift coefficient with experimental measurement [20] 


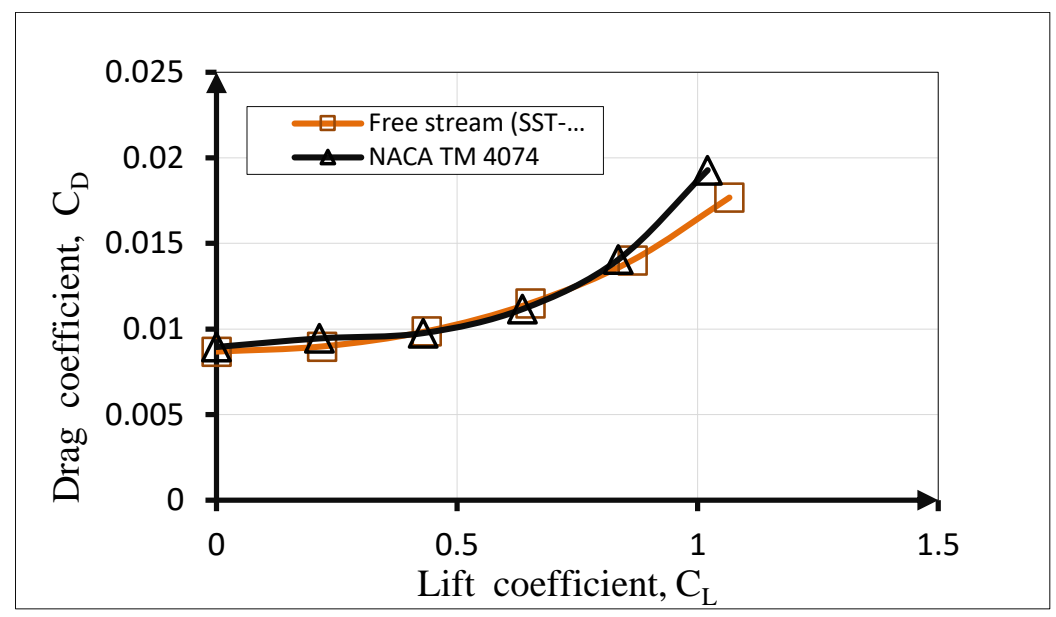

Fig. 8. Comparison of drag polar with experimental measurement $[21,22]$

\section{Comparison of Structured and Unstructured Mesh with Experimental Data}

In order to come up with a conclusion about the accuracy of the aerodynamic coefficients, the obtained coefficients for structured and unstructured grids are plotted in Figure 9 and Figure 10 together with the experimental measurements.

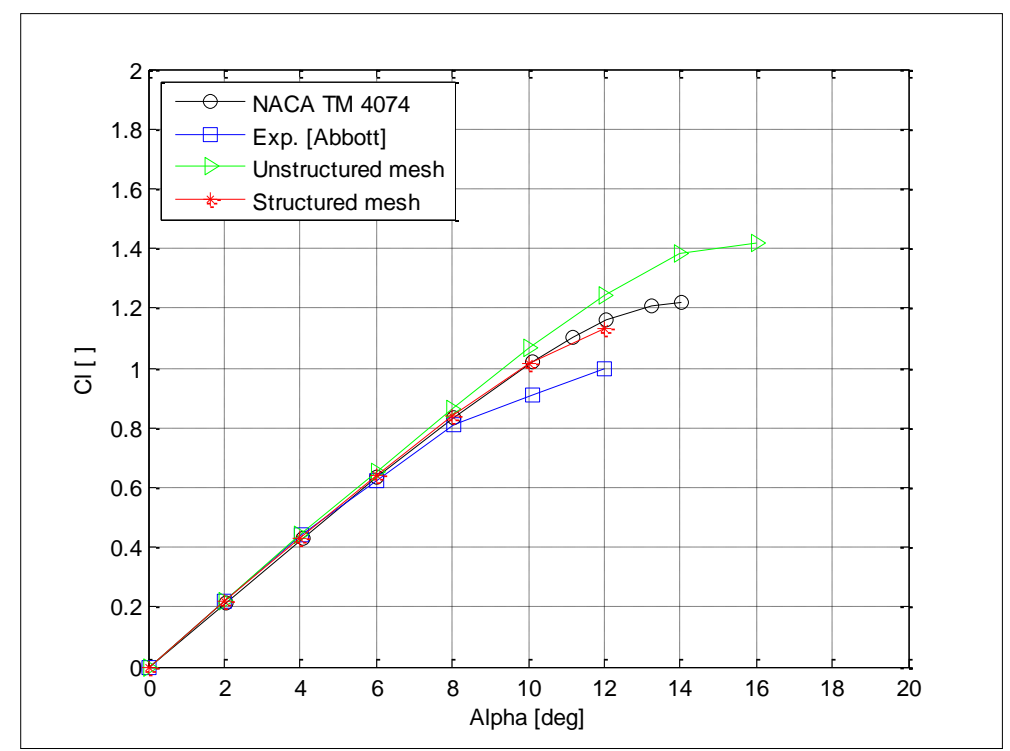

Fig. 9. Comparison of the lift coefficient curve between numerical and experimental data [20] 


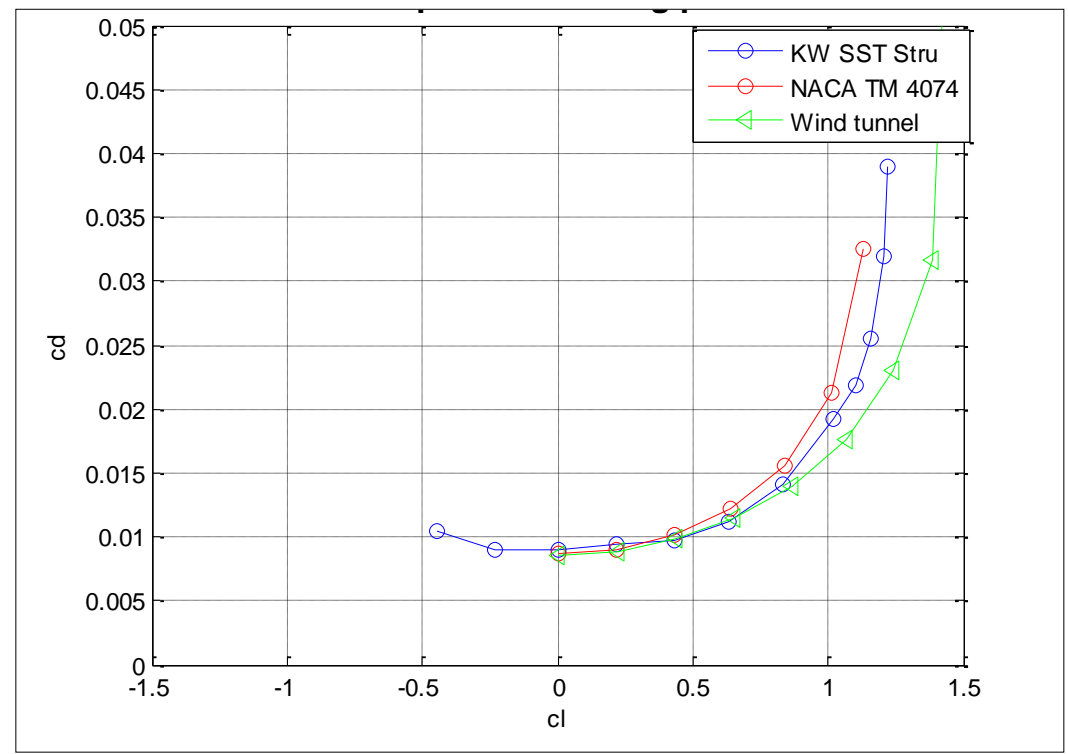

Fig. 10. Comparison between numerical and experimental data [21]

The above comparisons show that structured mesh results are closer to experimental data than unstructured mesh. In addition to that unstructured mesh consistently overestimates the lift coefficient, which results in a higher lift curve slope.

It is important to note that, generating a structured mesh with tilted airfoil with angles of attack more than 2 degrees was not possible with the prescribed domain shape. Due to this reason the unstructured grid was adopted to simulate wind tunnel wall effects on airfoil at all angles of attack. This is the topic of the next research paper.

\section{Conclusion}

In this paper, structured and unstructured meshes are generated for large computational domain 12 times the chord of NACA 0012 airfoil. A k- $\varpi$ SST turbulence model is used in all cases and angles of attack. The obtained lift and drag coefficients are compared at a Mach number of 0.15 and Reynolds number of $6 \times 10^{6}$ with available experimental measurements from the literature. The numerical results show that at the linear aerodynamic range, i.e. up to 8 degrees, structured and unstructured grid follow experimental data with the same accuracy. For angles of attack higher than 8 degrees, the unstructured mesh overestimates the experimental lift coefficient, while structured grid results are closer to experimental data. It is also worth mentioning that the experimental data of drag polar from both references show a clear discrepancy. The structure grid results lay in the middle of these experimental data.

\section{Declaration of Competing Interest}

The author(s) declared no potential conflicts of interest with respect to the research, authorship, and/or publication of this article.

\section{Acknowledgements}

The corresponding author acknowledge the Research Management Centre, (RMC), Universiti Tun Hussein Onn Malaysia for the financial support under the RMC Research Fund (H768). Authors are also very thankfull to the Modeling and Simulation Research Group, Department of Aeronautical Engineering, University of Zawia, Libya for consultation and support. 


\section{References}

[1] D'Alascio, A., Eurocopter Deutschland, K. Pahlke, and F. Le Chuiton. "Application of a structured and an unstructured CFD Method to the Fuselage Aerodynamics of the EC145 Helicopter. Prediction of the time averaged Influence of the Main Rotor." CD (2004).

[2] Wang, Tong, Zhixue Sun, and Jun Yao. "An efficient and robust fracture-grid and fracture-fracture intersection detection method for polygon fractures in unstructured polyhedral grids." Computers and Geotechnics 134 (2021): 104125. https://doi.org/10.1016/i.compgeo.2021.104125

[3] Badry, Ahmed Hassaani, Hesham M. El-Banna, Ahmed F. El-Sayed, and Galal B. Salem. "Computational Investigation (Simulation) of Flow Field and Aerodynamic Analysis Over NACA 23012 Airfoil With Leading Edge Glaze Ice Shape" Proceedings of ICFD11 (2013).

[4] Liu, Y. Y., Chang Shu, H. W. Zhang, and L. M. Yang. "A high order least square-based finite difference-finite volume method with lattice Boltzmann flux solver for simulation of incompressible flows on unstructured grids." Journal of Computational Physics 401 (2020): 109019. https://doi.org/10.1016/j.jcp.2019.109019

[5] Liu, Y. Y., H. W. Zhang, L. M. Yang, and C. Shu. "High-order least-square-based finite-difference-finite-volume method for simulation of incompressible thermal flows on arbitrary grids." Physical Review E 100, no. 6 (2019): 063308.

[6] Liu, Y. Y., C. Shu, H. W. Zhang, L. M. Yang, and Cunbiao Lee. "An efficient high-order least square-based finite difference-finite volume method for solution of compressible Navier-Stokes equations on unstructured grids." Computers \& Fluids 222 (2021): 104926. https://doi.org/10.1016/i.compfluid.2021.104926

[7] Khashan, Saud, and Abdullatif M. Alteraifi. "Steady-states versus time-accurate simulations for sub-critical flows around circular cylinder." COMPUTATIONAL FLUID DYNAMICS JOURNAL 13 (2004): 70-78.

[8] Elfaghi, A. M., W. Asrar, and A. A. Omar. "A High Order Compact-Flowfield Dependent Variation (HOC-FDV) Method for Inviscid Flows." International Journal for Computational Methods in Engineering Science and Mechanics 11, no. 5 (2010): 258-263. https://doi.org/10.1080/15502287.2010.501322

[9] Abdulhafid, M. Elfaghi, Asrar Waqar, and A. Omar Ashraf. "Accurate compact flowfield-dependent variation method for compressible euler equations." (2009).

[10] Mao, Zirui, and G. R. Liu. "A Lagrangian gradient smoothing method for solid-flow problems using simplicial mesh." International Journal for Numerical Methods in Engineering 113, no. 5 (2018): 858-890. https://doi.org/10.1002/nme.5639

[11] Mao, Zirui, G. R. Liu, Xiangwei Dong, and Tao Lin. "A conservative and consistent Lagrangian gradient smoothing method for simulating free surface flows in hydrodynamics." Computational Particle Mechanics 6, no. 4 (2019): 781-801. https://doi.org/10.1007/s40571-019-00262-z

[12] Mao, Zirui, G. R. Liu, and Yu Huang. "A local Lagrangian gradient smoothing method for fluids and fluid-like solids: A novel particle-like method." Engineering Analysis with Boundary Elements 107 (2019): 96-114. https://doi.org/10.1016/i.enganabound.2019.07.003

[13] Sohankar, Ahmad, Lars Davidson, and Christoffer Norberg. "Large eddy simulation of flow past a square cylinder: comparison of different subgrid scale models." J. Fluids Eng. 122, no. 1 (2000): 39-47. https://doi.org/10.1115/1.483224

[14] Voke, Peter R. "Flow past a square cylinder: test case LES2." In Direct and large-eddy simulation II, pp. 355-373. Springer, Dordrecht, 1997.

[15] Mao, Zirui, and G. R. Liu. "A 3D Lagrangian gradient smoothing method framework with an adaptable gradient smoothing domain-constructing algorithm for simulating large deformation free surface flows." International Journal for Numerical Methods in Engineering 121, no. 6 (2020): 1268-1296. https://doi.org/10.1002/nme.6265

[16] Abobaker, Mostafa, Abdulhafid M. Elfaghi, and Sogair Addeep. "Numerical Study of Wind-Tunnel Wall Effects on Lift and Drag Characteristics of NACA 0012 Airfoil." CFD Letters 12, no. 11 (2020): 72-82. https://doi.org/10.37934/cfdl.12.11.7282

[17] Khan, Sher Afghan, Musavir Bashir, Maughal Ahmed Ali Baig, and Fharukh Ahmed Ghasi Mehaboob Ali. "Comparing the Effect of Different Turbulence Models on The CFD Predictions of NACA0018 Airfoil Aerodynamics." CFD Letters 12, no. 3 (2020): 1-10. https://doi.org/10.37934/cfdl.12.3.110

[18] Ferziger, Joel H., and M. Perić. "Properties of numerical solution methods." Computational methods for fluid dynamics 3, no. 2 (2002): 31-35. https://doi.org/10.1007/978-3-642-56026-2

[19] Johnson, Richard W., ed. Handbook of fluid dynamics. Crc Press, 2016. https://doi.org/10.1201/b19031

[20] B. Allen, "NACA Airfoils". nasa.gov. NASA. Retrieved 24 June 2021.

[21] Ladson, Charles L. Effects of independent variation of Mach and Reynolds numbers on the low-speed aerodynamic characteristics of the NACA 0012 airfoil section. Vol. 4074. National Aeronautics and Space Administration, Scientific and Technical Information Division, 1988. 
[22] Sogukpinar, Haci. "The effects of NACA 0012 airfoil modification on aerodynamic performance improvement and obtaining high lift coefficient and post-stall airfoil." In AIP conference proceedings, vol. 1935, no. 1, p. 020001. AIP Publishing LLC, 2018. https://doi.org/10.1063/1.5025955 